\title{
On the Mechanism of Lead Chalcogenide Nanocrystal Formation
}

\author{
Jonathan S. Steckel, Brian K. H. Yen, David C. Oertel, and Moungi G. Bawendi
}

\section{Supporting Information}

General. Lead (II) acetate trihydrate (99.999\%), diphenylphosphine (>90\%), and anhydrous chloroform (>99\%) were purchased from Aldrich. Oleic acid (>99\%) was obtained from TCI America, octadecene (>95\%) from Fluka, tri-n-octylphosphine (97\%) from Strem, and selenium shot (99.999\%) from Alfa Aesar. All reagents were used as received.

All ${ }^{31} \mathrm{P}$ NMR spectra were acquired on a Varian Mercury $300 \mathrm{MHz}$ NMR spectrometer with an Oxford Instruments Ltd. superconducting magnet and a switchable probe $\left({ }^{1} \mathrm{H} /{ }^{19} \mathrm{~F} /{ }^{13} \mathrm{C} /{ }^{31} \mathrm{P}\right)$. For each spectrum, 256 transients were acquired and referenced to an external phosphoric acid standard. Flame atomic absorption spectroscopy (FAA) was performed using a Perkin-Elmer Analyst 300. Near-IR absorption data were acquired on a Cary 5000 UV-Vis-NIR spectrophotometer. Optical absorption spectra were recorded with an HP/Agilent 8453 diode array spectrometer. Electrospray mass spectrometry (ESI-MS) was performed using a $4.7 \mathrm{~T}$ Bruker ApexIV FT-ICR-MS with an Apolo ESI source. Fourier transform infrared (FT-IR) spectra were obtained using a Perkin-Elmer System 2000.

$\mathbf{P b}(\text { oleate })_{2}$ Characterization. $\mathrm{Pb}(\text { oleate })_{2}$ is produced when $\mathrm{Pb}(\mathrm{OAc})_{2} \cdot 3 \mathrm{H}_{2} \mathrm{O}$ and oleic acid are together heated under vacuum. The presence of acetic acid was confirmed by the odor in the vacuum trap and by analysis of trap contents using ${ }^{1} \mathrm{H}$ NMR. A number of solvents are commonly used in PbSe syntheses: diphenylether (DPE), octadecene (ODE), dioctylether (DOE), squalene, and tri-n-octylphosphine (TOP). When the degassed lead precursor solution is left at room temperature, a white waxy precipitate forms over time in all solvents except TOP. This white waxy precipitate was isolated from the solvent and was soluble in TOP, chloroform, dichloromethane, and toluene.

FT-IR spectroscopy, mass spectrometry, and elemental analysis confirmed the identity of the white waxy substance to be $\mathrm{Pb}$ (oleate) $)_{2}$. FT-IR spectra (sample film on PTFE card) recorded for oleic acid and the white waxy solid are shown in Figure S2. A broad O-H stretch at $3000 \mathrm{~cm}^{-1}$ and the $\mathrm{O}-\mathrm{H}$ out-of-plane bending at $934 \mathrm{~cm}^{-1}$ seen in the oleic acid spectrum are absent in the spectrum of the white solid. Also, the $\mathrm{C}=\mathrm{O}$ stretch disappears in the white solid spectrum while two new C-O stretches appear at 1513 and $1407 \mathrm{~cm}^{-1}$. When the head of a carboxylic acid is associated with a metal atom, the interaction is categorized as four types: monodentate, bridging bidentate, chelating bidentate, and ionic. The wavenumber separation $(\Delta)$ between the symmetric $v_{s}(\mathrm{COO}-)$ stretch and the asymmetric $v_{\text {as }}(\mathrm{COO}-)$ stretch IR bands can be used to diagnose the type of interaction. Large $\Delta\left(200-300 \mathrm{~cm}^{-1}\right)$ correspond to a monodentate interaction, medium $\Delta$ (140-190 $\left.\mathrm{cm}^{-1}\right)$ correspond to a bridging bidentate interaction, and small $\Delta\left(<110 \mathrm{~cm}^{-1}\right)$ correspond to a chelating bidentate interaction. ${ }^{1}$ Here we see a difference of $\Delta=1513-1407=$ $106 \mathrm{~cm}^{-1}$, corresponding to the chelating bidentate species as we would expect in lead oleate. ESI-MS calcd. $m / z$ for $\mathrm{C}_{36} \mathrm{H}_{66} \mathrm{O}_{4} \mathrm{PbH}^{+}\left(\left[\mathrm{M}+\mathrm{H}^{+}\right]\right):$771.5. Found: 771. Anal. calcd. for $\mathrm{C}_{36} \mathrm{H}_{66} \mathrm{O}_{4} \mathrm{~Pb}$ : C, 56.1; $\mathrm{H}, 8.6$; $\mathrm{Pb}, 26.9$. Found: $\mathrm{C}, 55.1 ; \mathrm{H}, 8.7 ; \mathrm{Pb}, 24.2$.

Investigation of mechanism involving $\mathrm{Se}^{2-}$. Tri- $n$-octylphosphine selenide (TOPSe, 2.24 M) was made by stirring excess elemental selenium shot in tri- $n$-octylphosphine (TOP) for several days. $0.5 \mathrm{mmol} \mathrm{Pb}(\mathrm{OAc})_{2} \cdot 3 \mathrm{H}_{2} \mathrm{O}$ and $1 \mathrm{mmol}$ oleic acid in $2.5 \mathrm{~mL}$ of octadecene were 
degassed under vacuum for 4 hours at $100^{\circ} \mathrm{C}$. During this time, $\mathrm{Pb}$ (oleate) $)_{2}$ formed, accompanied by the removal of acetic acid. $0.5 \mathrm{~mL}\left(0.089 \mathrm{mmol}\right.$ of $\left.\mathrm{Pb}(\text { oleate })_{2}\right)$ of this solution was put in each of four NMR tubes along with $37 \mu \mathrm{L} 2.24 \mathrm{M}$ TOPSe $(0.082 \mathrm{mmol}$ of Se) of. The four NMR tubes were sealed under nitrogen, the solutions mixed via tube inversion, and placed into an oil bath at $170^{\circ} \mathrm{C}$. The initially clear solutions quickly (after a couple of seconds) turned dark brown and then black over time as the PbSe nanocrystals (NCs) formed. The first tube was taken out of the oil bath after 10 minutes, the second after 20 minutes, the third after 30 minutes, and the fourth after 40 minutes. $0.3 \mathrm{~mL}$ of anhydrous chloroform was added to each tube after removal. (We found that the addition of chloroform further solvated tri- $n$ octylphosphine oxide (TOPO), providing sharper NMR peaks.) The ${ }^{31} \mathrm{P}$ NMR spectrum of each tube was acquired within an hour, followed by the isolation via centrifugation and complete digestion of the $\mathrm{PbSe} \mathrm{NCs}$ for reaction yield determination.

Investigation of mechanism involving $\mathrm{Se}^{0}$. The $\mathrm{Pb}$ (oleate $)_{2}$ solution was prepared as described above. 2.24 M TOPSe was doped with 30 mol percent diphenylphosphine (DPP) before being added to the lead solution in an NMR tube. $0.5 \mathrm{~mL}$ of the $\mathrm{Pb}$ (oleate $)_{2}$ solution $(0.089 \mathrm{mmol})$ and $40 \mu \mathrm{L}$ of the doped TOPSe solution $(0.089 \mathrm{mmol} \mathrm{Se}, 0.027 \mathrm{mmol}$ of DPP $)$ were mixed in an NMR tube, which was sealed and placed into an oil bath at $170^{\circ} \mathrm{C}$ for 10 minutes. The entire experiment, including heating, was performed inside a glove box. When the NMR tube was removed from the oil bath, $0.3 \mathrm{~mL}$ of anhydrous chloroform was added, the tube was sealed and removed from the glove box, and the ${ }^{31} \mathrm{P}$ NMR spectrum was acquired.

Batch synthesis modified with diphenylphosphine (DPP). $1 \mathrm{mmol}$ lead acetate trihydrate, $3 \mathrm{mmol}$ oleic acid, and $20 \mathrm{~mL}$ diphenylether were degassed under vacuum in a 50 -mL roundbottom flask at $71^{\circ} \mathrm{C}$ for 2 hours. $5 \mathrm{~mL} 1 \mathrm{M}$ TOPSe (either undoped or doped with 0.08 or 0.15 mmol DPP) was injected into the $\mathrm{Pb}$ (oleate) $)_{2}$ solution at $135^{\circ} \mathrm{C}$, followed by growth in which the temperature slowly increased towards the temperature of injection. After 2 minutes, the heating mantle was removed, allowing the solution to cool to room temperature. A representative absorption spectrum is shown in Figure S1.

Yield Determination. Reaction yields for our mechanism experiments, which were optimized for analysis with ${ }^{31} \mathrm{P}$ NMR and not NC quality, could not be calculated from the absorption spectra owing to poor size distributions and ill-defined first-absorption features. Yields were therefore determined by FAA spectroscopy. The NCs were first isolated from solution using centrifugation (6000 rpm). They were then digested using $\mathrm{HNO}_{3}$, and the solution was diluted with water for analysis

Batch syntheses modified by doping TOPSe with DPP resulted in monodisperse samples with well-defined absorption features. Reaction yields on these samples could be extracted directly from the NC absorption spectra as follows: First, a 2-3 mL portion of growth solution was removed and these NCs were precipitated twice with methanol and finally redispersed in 1,1,2-trichlorotrifluoroethane, which has no absorption features in the 1000-2000 nm window. An absorption spectrum was recorded, and from the position of the band-edge absorption peak, the NC diameter was determined. ${ }^{2}$ A small aliquot of growth solution was then diluted at a known factor in hexane, and the absorption at $400 \mathrm{~nm}$ was measured. Based on both calculated and experiment values for absorption cross section per $\mathrm{NC}$ at $400 \mathrm{~nm}$ (below), $\mathrm{NC}$ concentrations (and hence yields) were calculated. 
Absorption Cross-Section. The absorption cross-section at $400 \mathrm{~nm}$ was calculated using equation S1, based on Ref. 3 .

$$
C_{\text {abs }}=\frac{2 \pi}{m_{3} \lambda} 9 m_{3}^{4} \frac{2 n_{1} k_{1}}{\left(n_{1}^{2}-k_{1}^{2}+2 m_{3}^{2}\right)^{2}+4\left(n_{1} k_{1}\right)^{2}} \frac{4}{3} \pi a^{3}
$$

$C_{\text {abs }}$ is proportional to the product of the bulk semiconductor absorption coefficient $\left(2 n_{1} k_{1}\right)$ and a local field factor that is equal to the ratio of the applied electric field to the electric field inside the NC. The parameters $n$ and $k$ are components of the complex refractive index for bulk $\mathrm{PbSe},{ }^{4} m_{3}$ is refractive index of hexane, $\lambda$ is excitation wavelength, and $a$ is the $\mathrm{NC}$ radius. The calculated per particle absorption cross section (in $\mathrm{cm}^{2}$ ) for PbSe at $400 \mathrm{~nm}$ was found to be $C_{\text {abs }}$ $=\left(6.552 \times 10^{5}\right) a^{3} \mathrm{~cm}^{2}$.

We confirmed the validity of (S1) by directly measuring the single-exciton saturation curve of $4.5 \mathrm{~nm}$ diameter NCs. The NCs, dispersed in hexanes, were excited with a frequency doubled, regeneratively amplified titanium-sapphire laser (100 fs pulse width at $400 \mathrm{~nm}, 1 \mathrm{kHz}$ repetition rate). The photoluminescence spectra were collected (Princeton Instruments OMA V 512-pixel InGaAs array interfaced with an Acton 300i spectrometer) at various pump powers, yielding the saturation curve shown in Figure S3. Fitting the curve ${ }^{5}$ gives an experimentally determined value of $C_{\text {abs }}=1.05 \times 10^{-14} \mathrm{~cm}^{2}$ at $400 \mathrm{~nm}$. Substitution of the NC size into equation S1 gives a theoretical value of $C_{\mathrm{abs}}=7.46 \times 10^{-15} \mathrm{~cm}^{2}$, which is in good agreement with the experimental value.

\section{References}

1. N. Wu, L. Fu. M. Su, M. Aslam, K. C. Wong, V. P. Dravid, Nano Lett. 2004, 4, 383

2. J. S. Steckel et al, Adv. Mater. 2003, 15, 1862

3. C. A. Leatherdale et al, J. Phys. Chem. B 2002, 106, 7619

4. Handbook of Optical Constants of Solids, Academic Press, Inc., 1985, pg. 520.

5. J.-M. Caruge, Y. Chan, V. Sundar, H.J. Eisler, M.G. Bawendi, Phys. Rev. B 2004, 70, 085316. 


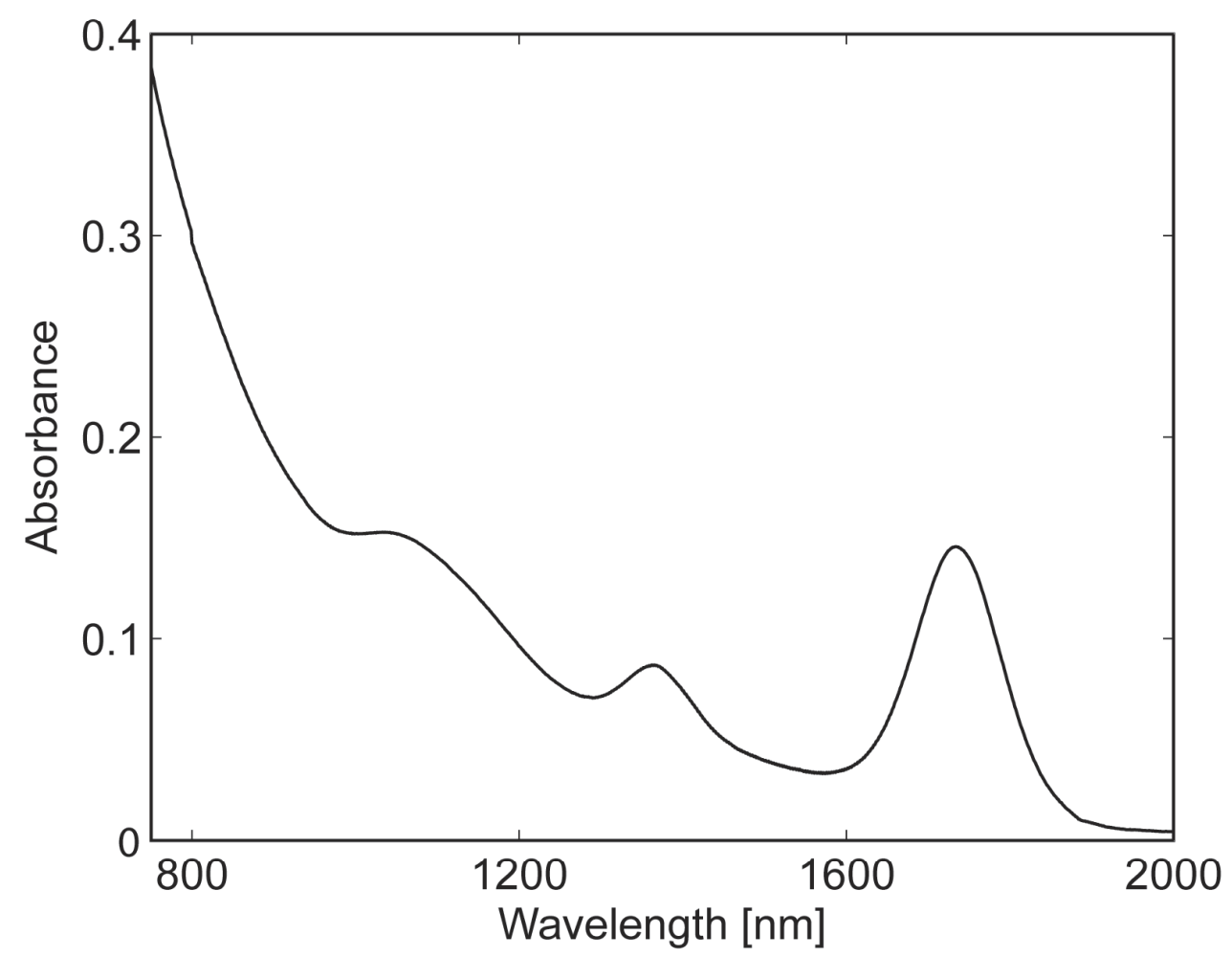

Figure S1. Representative absorption spectrum of PbSe NCs produced with batch synthesis using TOPSe doped with DPP (in this case, $0.08 \mathrm{mmol}$ ). 


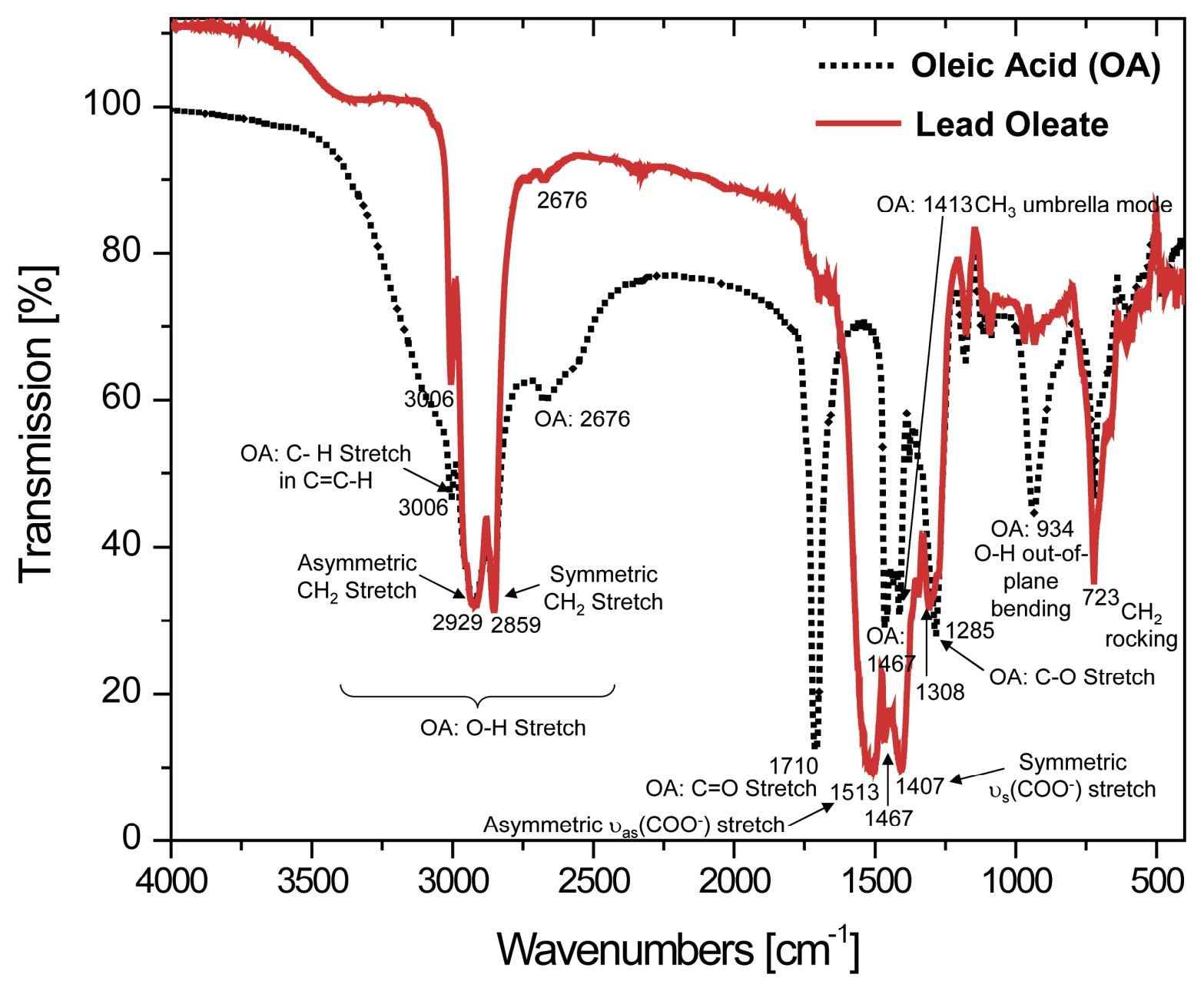

Figure S2. FT-IR spectra of oleic acid (black, dotted) and $\mathrm{Pb}\left(\right.$ oleate) ${ }_{2}$ (red, solid). 


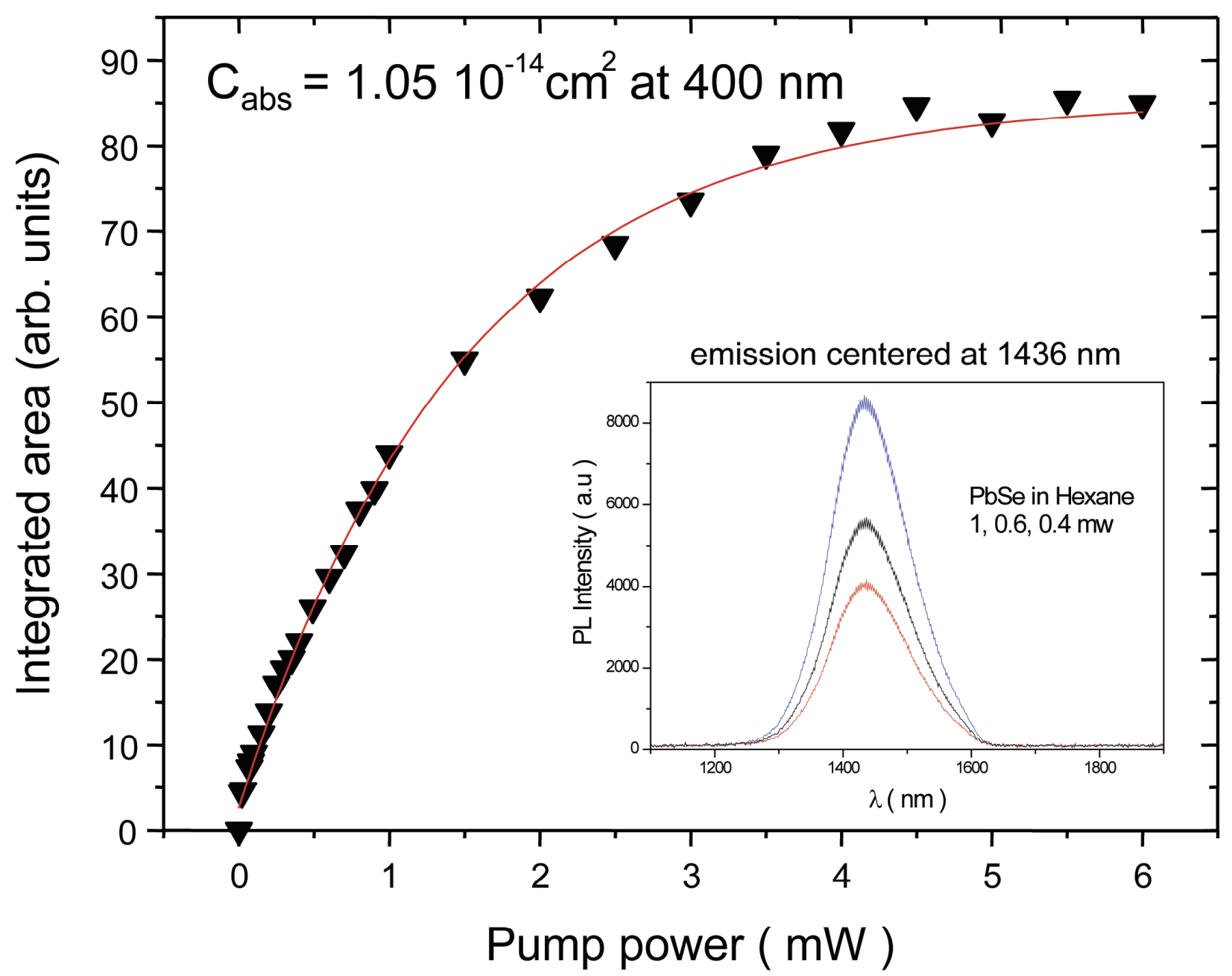

Figure S3. Single-exciton saturation curve of 4.5-nm PbSe NCs excited with 400-nm laser light of varying intensity. 